\title{
Whole-Genome Analysis of Coxsackievirus B3 Reflects Its Genetic Diversity in China and Worldwide
}

\section{Qian Yang}

National Institute for Viral Disease Control and Prevention https://orcid.org/0000-0001-9424-2956

Dongmei Yan

National Institute for Viral Disease Control and Prevention

\section{Yang Song}

National Institute for Viral Disease Control and Prevention

Shuangli Zhu

National Institute for Viral Disease Control and Prevention

Yun He

National Institute for Viral Disease Control and Prevention

Zhenzhi Han

National Institute for Viral Disease Control and Prevention

\section{Dongyan Wang}

National Institute for Viral Disease Control and Prevention

\section{Tianjiao Ji}

National Institute for Viral Disease Control and Prevention

Yong Zhang ( $\square$ yongzhang75@sina.com )

National Institute for Viral Disease Control and Prevention https://orcid.org/0000-0002-2692-5437

Wenbo Xu

National Institute for Viral Disease Control and Prevention

\section{Research}

Keywords: Coxsackievirus B3 (CVB3), Genome, Recombination lineages, Genetic diversity

Posted Date: November 15th, 2021

DOI: https://doi.org/10.21203/rs.3.rs-1038176/v1

License: (c) (1) This work is licensed under a Creative Commons Attribution 4.0 International License.

Read Full License 
Version of Record: A version of this preprint was published at Virology Journal on April 18th, 2022. See the published version at https://doi.org/10.1186/s12985-022-01796-0. 


\section{Abstract \\ Background}

Coxsackievirus B3 (CVB3) has emerged as an active pathogen in myocarditis, aseptic meningitis, hand, foot, and mouth disease (HFMD), and pancreatitis, and is a heavy burden on public health. However, CVB3 has not been systematically analyzed with regard to whole-genome diversity and recombination. Therefore, this study was undertaken to systematically examine the genetic characteristics of CVB3 based on its whole genome.

\section{Methods}

We combined CVB3 isolates from our national HFMD surveillance and global sequences retrieved from GenBank. Phylogenetic analysis was performed to examine the whole genome variety and recombination forms of CVB3 in China and worldwide.

\section{Results}

Phylogenetic analysis showed that CVB3 strains isolated worldwide could be classified into groups A-E based on the sequence of the entire VP1 region. The predominant CVB3 strains in China belonged to group D, whereas group E CVB3 might be circulated globally compared to other groups. The average nucleotide substitution rate in the $P 1$ region of CVB3 was $4.82 \times 10^{-3}$ substitutions/site/year. Myocarditis was more common with group A. Groups $C$ and $D$ presented more cases of acute flaccid paralysis, and group D may be more likely to cause HFMD. Multiple recombination events were detected among CVB3 variants, and there were twenty-three recombinant lineages of CVB3 circulating worldwide.

\section{Conclusions}

Overall, this study provides full-length genomic sequences of CVB3 isolates with a wide geographic distribution over a long-term time scale in China, which will be helpful for understanding the evolution of this pathogen. Simultaneously, continuous surveillance of CVB3 is indispensable to determine its genetic diversity in China as well as worldwide.

\section{Background}

Enteroviruses (EVs) are important etiological agents that can cause a wide spectrum of diseases in young children, ranging from febrile illness, hand, foot, and mouth disease (HFMD), herpangina, encephalitis, acute flaccid paralysis (AFP), and even death [1-4]. EVs belong to the genus Enterovirus in the family Picornaviridae and order Picornavirales. Human EVs comprise four species, namely, EV-A, EV-B, EV-C, and EV-D [1-4]. To date, EV-B includes 63 serotypes, including the coxsackievirus group A (CVA, 
serotypes 9), coxsackievirus group B (CVB, serotypes 1-6), echovirus (serotypes 1-7, 9, 11-21, 24-27, and 29-33), EV-B69, EV-B73-B75, EV-B77-B88, EV-B93, EV-B97-B98, EV-B100-B101, EV-B106-B107, and EV-B111; and non-human EVs: EV-B110, EV-B112-114 [5].

Coxsackievirus B3 (CVB3) was first reported in Connecticut, USA in 1949, and has emerged as an active pathogen in myocarditis [6], aseptic meningitis (AM), $\operatorname{HFMD~[7,~8],~and~pancreatitis~[9],~causing~a~heavy~}$ burden on society. CVB3 infection is the most common cause of viral myocarditis, characterized by viral infection and myocardial inflammation $[10,11]$. However, there are no vaccines or antiviral drugs available to treat CVB3 infections.

Generally, EVs were considered to have only one ORF, but a second ORF was characterized in the EV genome and its encoded protein, ORF2p [12], was found to be crucial for viral intestinal infection. In CVB3, this ORF was found to be span $\mathrm{ATG}^{589} \mathrm{nt}$ to $\mathrm{TGA}^{792} \mathrm{nt}$ in the prototype strain (Nancy). The first ORF encodes 11 proteins, including VP1 to VP4, 2A to $2 \mathrm{C}$, and 3A to 3D. The amino acid sequence of the ORF2 product, ORF2 $p$, is highly conserved among various EVs (EV-A, $B$, and $C)$, suggesting an important role for ORF2p in EV replication or transmission.

HFMD is a viral infectious disease in young children, particularly in those younger than 5 years of age, and is associated with different types of EVs, notably enterovirus A71 (EV-A71) and Coxsackievirus A16 (CVA16) [13-15]. However, non-EV-A71 and non-CVA16 EVs became the dominant pathogens in the HFMD spectrum from 2013 in mainland China [1, 3]. Similarly, CVB3 is becoming a vital pathogen in the HFMD spectrum [8]. The prevalence of HFMD caused by CVB3 also increases the risk of serious diseases such as myocarditis.

Frequent recombination events and mutations in EVs have been recognized as the main mechanisms for the high rate of evolution observed, which enables them to rapidly respond and adapt to new environmental challenges [16]. Recombination is a frequent inter- and intra-typic event in EVS and is an important cause for the emergence of new EV lineages and types [17].

Relying on our three-level HFMD surveillance laboratory network in China, we obtained temporal and geographical representative CVB3 sequences isolated from patients with HFMD and from healthy children. To date, there has been no systematic analysis of the whole-genome variety and recombination forms of CVB3 in China and worldwide. Therefore, we performed a large-scale, (near) full-length genetic analysis of global and Chinese CVB3 isolates, including 26 newly sequenced samples collected extensively in mainland of China between 2012 and 2020 CVB3 sequences retrieved from the GenBank database.

\section{Methods}

\section{Virus isolation}


Clinical specimens were collected from patients with HFMD and from healthy children in mainland of China. Real-time reverse transcription-polymerase chain reaction (RT-PCR) was performed to screen for EV-A71, CVA16, and other EVs as previously described [10]. Non-EV-A71 and non-CVA16 EV-positive samples were selected for further analysis. All samples were processed according to the standard and previously described protocols [9]. The viruses were isolated from the original clinical specimens by propagation in human rhabdomyosarcoma (RD) and human larynx carcinoma (HEp-2) cells following conventional methods [11]. These two cell lines were obtained from the WHO Global Poliovirus Specialized Laboratory, USA, and were originally purchased from the American Type Culture Collection.

\section{Full-length genome sequencing of CVB3 isolates}

We selected the non-EV-A71 and non-CVA16 EV-positive samples, and extracted the viral RNA using a QIAamp Viral RNA Mini Kit (Qiagen, Valencia, CA, USA). Reverse transcription polymerase chain reaction (RT-PCR) was performed to amplify the entire VP1 capsid region using the PrimeScript One Step RT-PCR Kit Ver. 2 (TaKaRa, Dalian, China) and the primers E490/E492 were used to amplify a portion of the VP1 region [8]. Preparation of RT-PCR reactions and amplification profiles were based on previously reported protocols [8]. PCR products were purified using a QIAquick PCR Purification Kit (Qiagen, Germany). The amplicons were bi-directionally sequenced using an ABI 3130 Genetic Analyzer (Applied Biosystems, USA). Sequences were analyzed using the EV Genotyping Tool and BLAST server [18]. To obtain the entire genome sequence of CVB3, specific primers were used based on a previous study [8]. The obtained sequences were deposited in GenBank under the accession numbers OK632332-OK632353, OK643871OK643874, OK643877-OK643882. The CVB3 strains used in this study were isolated between 2012 and 2020. Twenty-six samples isolated between 2012 and 2020 were identified as CVB3. The geographic distribution map of CVB3 isolates from mainland China was obtained from Highcharts (Grant number: 0321912045738052).

\section{Phylogenetic analysis of CVB3 sequences}

Alignment of the nucleotide sequences from CVB3 strains was performed using the BioEdit sequence alignment editor software (version 5.0). Maximum likelihood (ML) trees were estimated using the best-fit Tamura-Nei model (TN93) + Gamma (G) parameter model of nucleotide substitution in MEGA software (version 5.03). Branch lengths of the dendrogram were determined based on the tree topology and the majority rule consensus of 1,000 bootstrap replicates. Bootstrap values greater than $70 \%$ were considered statistically significant for grouping. The global evolutionary dynamics of CVB3 were inferred based on the entire $P 1$ capsid region. The correlation coefficient and regression value of each dataset were calculated using TempEst ( $v$ 1.5.1) to estimate the correlation between sequence divergence and the date of isolation in each dataset [19]. The Markov chain Monte Carlo (MCMC) method implemented in BEAST (v1.7.5) was used to estimate the temporal phylogenies and rates of evolution [20]. All $84 P 1$ region sequences were analyzed using the uncorrected relaxed molecular clock (exponential) and constant site tree prior to the $\mathrm{GTR}+\mathrm{G}+\mathrm{I}$ nucleotide substitution model. A Bayesian MCMC run comprised $3 \times 10^{8}$ generations to ensure that each parameter could converge. The sampling frequency was set to 
$3 \times 10^{4}$ generations. The output from BEAST was analyzed using TRACER (v1.7.1)

(http://beast.community/tracer) (with estimated sample size (ESS) values higher than 200). A maximum clade credibility (MCC) tree was constructed using TreeAnnotator, and the burn-in option was used to remove the first $10 \%$ of the sampled trees; the resulting tree was visualized using FigTree (v1.4.4).

\section{Recombination analysis of CVB3 sequences}

In this study, we scanned the entire genomic sequence of CVB3 and potential recombinants from GenBank. Briefly, the $P 2$ and $P 3$ coding sequences of these strains were analyzed using BLAST to compare their identity with the sequences from GenBank. Sequences with a similarity higher than 85 percent were considered potential parental sequences and were downloaded from GenBank. Similarity plots and bootscanning analyses were performed using Simplot (version 3.5.1; Stuart Ray, Johns Hopkins University, Baltimore, MD, USA). A sliding window of 200 nucleotides was used, moving in 20-nucleotide steps, and bootscanning analyses were performed using the neighbor-joining method [21].

\section{Results}

\section{Global CVB3 epidemiology}

With the rapid development of molecular biology technology, a cDNA copy covering two-thirds of the CVB3 genome was cloned in Sweden in 1984 [22]. In 1985, the biologically active CVB3 virus (Nancy strain) CDNA, including the entire genome, was synthesized in Germany [23]. When the full-length genome of CVB3 was first reported in 1987, the number of CVB3 isolates significantly increased. CVB3 related outbreaks have been reported frequently, such as an outbreak in southern Louisiana, USA in 1959 (51 patients) [24], an outbreak in South Africa in 1984 with a variety of clinical manifestations [25], an outbreak of herpangina in Japan in 1987[26] (22 patients), an outbreak in Thailand in 1988 [27], an October 1992 outbreak of infant infection in a hospital in Beijing (35 patients) [28], a June 2005 outbreak of neonatal infections in Taiwan, China [29], 2008 outbreaks of aseptic meningitis in Shandong Province, China (81 CVB3 isolates) [30], and Hong kong, China (69 CVB3 isolates) [31], an outbreak of HFMD in Hebei in 2012 (35 cases), and an outbreak of HFMD in Shandong in 2016 (42 patients) [8]. During the EVD68 outbreak in 2014 in the United States, CVB3 was the most commonly identified type of EV infection [32].

To date, CVB3 strains are associated with various diseases, and the manifestations vary from mild respiratory [33], gastrointestinal infections [34, 35], herpangina [36], and hand, foot, and mouth disease [7, 8, 37], to more severe diseases such as heart disease (including viral myocarditis [6, 38], pericarditis [39], and acute myocardial infarction [40]), CNS involvement (meningitis [30, 36, 41-43], meningo-cerebellitis [11], encephalitis [44], and AFP [45-47]), and pancreatic interrelated disease (pancreatitis [48], diabetes [49]). More seriously, the virus can cause neonatal sepsis-like illness and sudden death in infected infants $[38,50]$ and adults with low immune function. 


\section{Summary Of Cvb3 Datasets}

In total, 319 entire VP1 sequences (before 2021 August 31th) were collected for the CVB3 genotyping and clinical manifestations summary. The CVB3 strains isolated between 1949 (the prototype strains) and 2020 from 17 countries, included countries in Asia (China, Japan, India, Thailand, Uzbekistan, Indonesia), Europe (Germany, France, UK, Poland, Denmark, Russia, Romania, Moldova), North America (USA), Oceania (Australia), and Africa (Madagascar), representing wide temporal and regional distributions. In total, 84 CVB3 whole genome sequences (near the whole genome) (this study, $n=26$; GenBank, $n=58$ ) were used for the analysis. The sequence information is summarized in Additional file 1: Table S1. In total, 44 CVB3 sequences were included from mainland of China from 2006 to 2020, in 17 provinces, municipalities, and autonomous regions, representing seven administrative regions of China: Southeast China (Shandong, Jiangsu, Shanghai, Anhui, and Fujian), Central China (Hunan), South China (Guangdong), North China (Beijing and Tianjin), Northwest China (Shaanxi, Gansu, and Xinjiang), Southwest China (Sichuan, Yunnan, and Tibet), and Northeast China (Jilin) (Fig. 1). CVB3 strains isolated between 1949 (the prototype strains) and 2020 were from 11 countries and regions, including mainland of China.

\section{Five groups were assigned based on the complete VP1 coding region of CVB3 isolates}

In total, 319 entire VP1 sequences were collected from mainland China $(n=167)$, Taiwan, China $(n=46)$, Japan $(n=3)$, India $(n=17)$, Thailand $(n=2)$, Uzbekistan $(n=1)$, Indonesia $(n=1)$, Germany $(n=27)$, France $(n=19)$, UK $(n=1)$, Poland $(n=4)$, Denmark $(n=1)$, Russia $(n=3)$, Romania $(n=2)$, Moldova $(n=$ $1)$, USA $(n=8)$, Australia $(n=14)$, and Madagascar $(n=2)$. We divided the global CVB3 into five groups (A-E) based on the entire VP1 sequence (Fig. 2A). The group mean distances varied from $16.9 \%$ (groups $C$ to $D$ ) to $21.5 \%$ (groups $A$ to $C$ ). The mean genetic distance within the group varied from $0.6 \%$ (group $A$ ) to $12.0 \%$ (group E), indicating the reliability of genotyping. The prototype strain Nancy, which was isolated in the USA in 1949, clustered with the strains isolated in Australia, the USA, Germany, and mainland of China to form Group A. Group B included strains isolated in the USA in 1956 and in Germany in 1999. Most Indian strains, one strain from Uzbekistan, and two from Madagascar formed group C.

Most Group D strains were isolated in China. Group E comprised strains isolated from 13 countries, suggesting that group E might be transmitted globally. Notably, the Chinese isolates fell into groups A, D, and E. Group D CVB3 was first isolated from cases of acute flaccid paralysis in 1990 and has persistently circulated in mainland of China from 1993 to 2017, suggesting strong transmission potential. We found that the dominant group D had a wide temporal and geographical distribution, whereas group $\mathrm{A}$ disappeared after 2014. Emerging group E was only detected among HFMD cases in 2020 in one province and was possibly imported from other countries. The basic data for each group are summarized in Table 1. 
Table 1

Information of 5 group CVB3s relying on entire VP1 sequences

\begin{tabular}{|c|c|c|c|c|}
\hline Group & $\begin{array}{l}\text { Sequences } \\
\text { number }\end{array}$ & Country of isolation & Year & $\begin{array}{l}\text { Intra-group } \\
\text { distances(\%) }\end{array}$ \\
\hline A & 12 & Australia, USA, Germany, China & $\begin{array}{l}1949- \\
2014\end{array}$ & 0.6 \\
\hline B & 2 & USA, Germany & $\begin{array}{l}1956- \\
1999\end{array}$ & 3.6 \\
\hline C & 20 & India, Uzbekistan, Madagascar & $\begin{array}{l}1999- \\
2011\end{array}$ & 5.2 \\
\hline D & 204 & China, Thailand, Russia, Japan, France & $\begin{array}{l}1990- \\
2017\end{array}$ & 8.3 \\
\hline$E$ & 81 & $\begin{array}{l}\text { China, Thailand, Indonesia, Japan, Moldova, Russia, } \\
\text { Romania, Poland, Germany, France, Denmark, the } \\
\text { UK, Australia, USA }\end{array}$ & $\begin{array}{l}1989- \\
2020\end{array}$ & 12.0 \\
\hline
\end{tabular}

To investigate the evolutionary history of CVB3, MCC trees (Fig. 2B) were constructed based on the entire $P 1$ nucleotide sequence of CVB3 strains $(n=77)$. The correlation coefficient was 0.74 and the R2 value was 0.54 . A positive correlation was observed between genetic divergence and sampling time. The average nucleotide substitution rate for the $P 1$ coding region in all CVB3 strains worldwide was $4.82 \times$

$10^{-3}$ substitutions/site/year ( $95 \% \mathrm{HPD}, 3.51 \times 10^{-3}-6.05 \times 10^{-3}$ ), which is slightly higher compared to the $P 1$ evolutionary rate of $\mathrm{CV}-\mathrm{A} 6$ reported by others [1], indicating its rapid spreading rate. The topological structure of the MCC tree constructed using BEAST was nearly identical to that of the ML tree constructed using MEGA software. Based on our analysis, the estimated T MRCA of CVB3 was in the early 1900s, corresponding to at least 40 years before the first reported detection of CVB3 in 1949. Global CVB3 strains isolated since 1903 formed two branches. Branch 1 included only group A, which arose in 1946, including the prototype strain Nancy. Branch 2 included group B (differentiated in 1927), group $C$ with a tMRCA that emerged in 1990, group D (emerged in approximately 1994), and group E emerging in 1982.

We also focused on the clinical manifestations of different groups. Myocarditis (41.7\%) was more common in group A than that in the other groups. Group C (40\%) and group D (31.4\%) presented more cases of AFP compared to the other groups. In addition, while comparing the numbers $(n=65)$ and proportions (31.9\%) of HFMD cases among the five groups in this study, we found that group $D$ may be more likely to cause HFMD. The most common diseases in group E were HFMD (9.9\%), diabetes (9.9\%), and aseptic meningitis (8.6\%) (Fig. 2C).

\section{Full-length genome analysis of CVB3 exhibited diverse recombination lineages in China and worldwide}


Phylogenetic trees were constructed based on the entire $P 1, P 2$, and $P 3$ region nucleotide sequences of CVB3 strains along with the prototype of EV-B from GenBank database. Consistent with the phylogenetic tree of the VP1 coding region, the $P 1$ phylogenetic tree (Fig. 3A) indicated the existence of the five CVB3 groups in the world as expected, verifying the primary genotyping results. Unlike the $P 1$ phylogenetic trees, those of the $P 2$ and $P 3$ non-structural regions showed that several independent lineages clustered with the prototype strains of other EV-B prototypes rather than with the prototype of CVB3, suggesting the occurrence of recombination between CVB3 and other EV-B serotypes (Fig. 3B, 3C). The CVB3 whole genomes displayed significant genetic diversity in the non-structural regions, and a total of twenty-three recombinant lineages were detected (Table 2). We named the recombinant lineages based on their first isolation time. Groups A and B had only one lineage each, consistent with the VP1 region phylogenic tree. Group C contained three lineages: lineage $F$, lineage 01 , and lineage 02 . Group D isolates were differentiated into lineage $G$, lineage $K$, lineage $L$, lineage $M$, lineage $R$, lineage $S 1$, lineage $S 2$, lineage $T$, and lineage $U$, suggesting that at least nine different CVB3 recombinant lineages are circulating in China. Group E was separated into independent lineages by other EV-B prototype strains, including lineages C, D, $E, H, I, J, N, P$, and Q. Some recombinant lineages, such as lineages B-F, are ancient lineages from the last century and tend to be absent. The recombinant lineage $\mathrm{J}$ was associated with group $\mathrm{E}$, which clearly covered several countries and was spread extensively. We also used similarity scanning to certify the twenty-three diverse recombinant lineages using ORF1 sequences (Fig. 3D). The intra-lineage nucleotide similarity of lineages $A, I, J, K, L, 01,02, R, S, U$, and T was between 95.8-99.9\%, indicating that all the strains in the same lineage displayed high intra-lineage homology throughout the ORF1 sequences. The sliding window analysis performed across intra-lineage sequences revealed low pairwise genetic diversity; however, when all the lineages were considered together, the diversity was obviously higher in the non-capsid regions (Fig. 3E).

Table 2 Information of 23 recombinant lineages of CVB3s based on $P 2$ and $P 3$ non-structural region sequences 


\begin{tabular}{|c|c|c|c|c|c|}
\hline Lineage & Group & $\begin{array}{l}\text { Sequence } \\
\text { number }\end{array}$ & $\begin{array}{l}\text { Nucleotide mean } \\
\text { distances(\%) }\end{array}$ & $\begin{array}{l}\text { Isolated } \\
\text { countries/regions }\end{array}$ & $\begin{array}{l}\text { Isolated } \\
\text { year }\end{array}$ \\
\hline A & A & 10 & 0.46 & USA, China & $\begin{array}{l}1949- \\
2014\end{array}$ \\
\hline B & B & 1 & NA & USA & 1956 \\
\hline C & E & 1 & NA & Romania & 1989 \\
\hline D & E & 1 & NA & France & 1993 \\
\hline$E$ & E & 1 & NA & Romania & 1995 \\
\hline$F$ & C & 1 & NA & Uzbekistan & 1999 \\
\hline G & D & 1 & NA & China & 2001 \\
\hline $\mathrm{H}$ & $E$ & 1 & NA & Moldova & 2002 \\
\hline I & $E$ & 1 & NA & USA & 2005 \\
\hline J & $E$ & 11 & 4.14 & $\begin{array}{l}\text { Australia, the UK, USA, } \\
\text { China }\end{array}$ & $\begin{array}{l}2006- \\
2020\end{array}$ \\
\hline K & D & 9 & 4.24 & China & $\begin{array}{l}2006- \\
2013\end{array}$ \\
\hline L & D & 10 & 2.80 & China, Thailand & $\begin{array}{l}2008- \\
2012\end{array}$ \\
\hline M & D & 1 & NA & China & 2009 \\
\hline$N$ & E & 1 & NA & Australia & 2009 \\
\hline 01 & C & 11 & 1.39 & India & 2009 \\
\hline 02 & C & 1 & NA & India & 2009 \\
\hline$P$ & E & 1 & NA & Thailand & 2010 \\
\hline Q & E & 1 & NA & Denmark & 2010 \\
\hline $\mathrm{R}$ & D & 8 & 2.65 & China & $\begin{array}{l}2012- \\
2016\end{array}$ \\
\hline S1 & D & 2 & 0.10 & China & 2016 \\
\hline S2 & D & 2 & 0.43 & China & 2016 \\
\hline $\mathrm{T}$ & D & 2 & 1.84 & China & 2016 \\
\hline U & D & 6 & 2.30 & China & $\begin{array}{l}2016- \\
2017\end{array}$ \\
\hline
\end{tabular}


Previous studies have shown that EV-Bs are more susceptible to recombination compared to EV-As [51]. Regarding CVB3, many recombinant lineages, which have persisted for many years and were widespread, are still globally active. For example, lineage $J$ included 11 strains showing high sequence identity with each other. The $P 2$ and $P 3$ non-structural coding region of lineage $\mathrm{J}$, which clustered with the E18 (MG720260) and E30 (EF066392) strains, exhibit recombination activities in the evolutionary process of CVB3 (Fig. 4A). Furthermore, the non-structural genomic region of lineage $L$ was found to have recombination breakpoints with three different EV-B serotypes: CVB5 (MN749140, JX843811, JN695051), E25 (KJ957190), and E30 (KF878942) strains; in contrast, the $P 1$ coding region showed higher similarity with the CVB3 prototype strain (Fig. 4B). Lineage 01 showed a complex recombination with the E11 (MK791152), E25 (MF678298), EVB75 (MW183139), and EVB88 (MH144607, MH118025) strains in the whole $P 3$ region (Fig. 4C). Lineage $U$ showed obvious recombination events, corresponding to the CVB5 (JN695051), E25 (KJ957190), and E30 (KF878942) strains (Fig. 4D). Therefore, the other serotypes of EVBs, such as echovirus (E11, E18, E25, and E30), CVB5, and novel EV-B75 and EV-B88 play important roles in the natural recombination of CVB3. These results indicate that inter-serotype recombination events occurred in the non-structural coding region of CVB3. The current circulating CVB3 strains are recombinants; however, the exact source of recombinant fragments was not identified; therefore, further studies are still needed to determine the EV serotype circulating simultaneously.

\section{Discussion}

In addition to EV-A71, CVA16, CVA6, and CVA10, other EVs also account for sporadic cases of HFMD and occasional outbreak events. CVB3, which was previously detected infrequently, was recently identified as a pathogen causing HFMD outbreaks [7, 8]. Persistent outbreaks of HFMD have been causing emerging nationwide epidemics since 2007; therefore, systematic national EV surveillance is needed to comprehensively study EV pathogens. With our continuous surveillance data on CVB3-related HFMD cases collected over a 10-year period, we performed a systematic analysis of CVB3 full-length genomic characteristics and recombination forms.

EVs are characterized by their high evolutionary rates, which are largely attributed to the low fidelity of replication with error-prone RNA polymerases. The evolutionary mechanisms of EVs include mutation and recombination. Genetic mutations can change the pathogenicity, antigenicity, and host range; further, recombination occurs extremely frequently, allowing independent evolution of EV genome fragments [52].

CVB3 can cause various human diseases, implying multiple strains with different virulences in a single serotype. Previous studies on CVB3 with clinical descriptions indicated a potential correlation between clinical phenotypes and genetic mutations. Nucleotide substitutions in the coding region and non-coding region are reported to be related to the virulence of CVB3, and can change the degree of damage to the organs. One study identified two differences among nucleotides in untranslated regions and 8 amino acid differences in the coding regions relative to the cardiovirulent CVB3 strains. It is reported that the mutation of amino acids in P126M and D155G in the VP1 region may lead to the attenuation of CVB3 without myocardial injury [53]. Other data indicate that amino acids at positions 124-241 of VP1 in CVB3 
are very important in protecting mice from death [52]. Thus, CVB3 strains can be divided into cardiovirulent strains (that are pathogenic for both the murine heart and the pancreas), pancreovirulent strains (that are pathogenic for the pancreas), and non-pathogenic strains. We compared the nucleotide and amino acid mutations across the entire VP1 region in different groups. The results showed that the amino acid positions of $110\left(X^{2}=449.45, P<0.001\right)$ and $223\left(X^{2}=124.54, P<0.001\right)$ were significantly different among the five groups (Fig. 5). Whether the two mutations are correlated with different types of diseases among different groups requires further experimental research.

Intratypic and intertypic forms of homologous recombination are also thought to be relatively frequent evolutionary forces that affect enteroviral genomes. Although the number of whole genomes was limited, we could still find the recombination diversity in global CVB3 sequences. We allocated the worldwide CVB3 variants to twenty-three different evolutionary recombinant lineages based on non-capsid sequences. Several previous studies showed that Chinese CVB3 showed the highest similarity with the parental CVB3 in the $P 1$ region, with the E25 strain in the $P 2-P 3$ region, and other strains of CVB3 showed similarity with CVB5 in the $P 3$ region $[54,55]$, which is consistent with our study. However, it remains unclear whether there are substantial differences in recombination frequencies between different $E V s$ such as echoviruses, and whether CVB5 plays a major role in the natural recombination of CVB3.

Previous studies on EVs, such as CVA6 [1], indicated a potential correlation between clinical phenotypes and recombination. Our research does have some limitations, as there is a limited number of CVB3 sequences in each lineage, and 13 lineages have only one sequence. Therefore, we could not analyze the potential correlation between the pathogenicity and genetic recombination of CVB3.

\section{Conclusions}

Overall, this study summarized the global CVB3 genetic diversity in both the VP1 region and the whole genome, and the database provided insight into the global phylogenetic characteristics of CVB3. The complete genome analysis provided more comprehensive and detailed information compared to that based only on the VP1 region. With more than half a century's circulation, different groups CVB3 had caused several outbreaks. Our results indicated a potential correlation between clinical phenotypes and genetic diversity. Therefore, continued surveillance is necessary to understand the complete genetic diversity of CVB3 in mainland China as well as worldwide.

\section{List Of Abbreviations}

enteroviruse: $\mathrm{EV}$

hand, foot, and mouth disease: HFMD

acute flaccid paralysis: AFP

coxsackievirus B3: CVB3

Page $12 / 23$ 
aseptic meningitis: AM

Real-time reverse transcription-polymerase chain reaction: RT-PCR

Rhabdomyosarcoma: RD

human larynx carcinoma: HEp-2

Markov chain Monte Carlo: MCMC

maximum clade credibility: MCC

\section{Declarations}

\section{Ethics approval and consent to participate}

The present study did not involve human participants or human experiments. The only human-derived materials used were stool samples, throat swab samples, and rectal swab samples collected from patients with HFMD for public health purposes under the programs of the Ministry of Health, P. R. of China. Written informed consent for the use of clinical samples was obtained from the parents of the children enrolled in the study. This study was approved by the Ethics Review Committee (IVDC2016-004, February 2016) of the National Institute for Viral Disease Control and Prevention (IVDC), Chinese Center for Disease Control and Prevention. All experimental protocols were approved by the IVDC, and the procedures were performed in accordance with approved guidelines.

\section{Consent for publication}

Not applicable

\section{Availability of data and materials}

The CVB3 nucleotide sequences determined in this study have been deposited in the GenBank nucleotide sequence database under accession numbers OK632332- OK632353, OK643871-OK643874, OK643877-0K643882.

\section{Competing interests}

The authors declare that they have no competing interests.

\section{Funding}

This study was supported by the National key research and development project (Project No. 2021YFC0863000) and the National Science and Technology Major Project of China (No.2018ZX10305409-004-002). The funding bodies were not involved in the design of the study, clinical 
sample collection, data analysis, or interpretation. They were also not involved in the writing of the manuscript or in the decision to publish the results.

\section{Authors' contributions}

YZ and WBX conceived and designed the experiments. QY, DMY, YS, SLZ, YH, ZZH, DYW, and TJJ performed the experiments. QY analyzed the data. QY, YZ and WBX wrote the main manuscript text. All authors reviewed the manuscript.

\section{Acknowledgements}

We would like to acknowledge the staffs in provincial center for disease control and prevention (CDC) for collecting specimens in this study.

\section{Author's information}

WHO WPRO Regional Polio Reference Laboratory, National Health Commission Key Laboratory for Medical Virology, National Institute for Viral Disease Control and Prevention, Chinese Center for Disease Control and Prevention, 155 Changbai Road, Beijing 102206, People's Republic of China

Qian Yang, Dongmei Yan, Yang Song, Shuangli Zhu, Yun He, Zhenzhi Han, Dongyan Wang, Tianjiao Ji, Yong Zhang, Wenbo Xu

Center for Biosafety Mega-Science, Chinese Academy of Sciences, Wuhan 430071, People's Republic of China

Yong Zhang, Wenbo Xu

Shandong First Medical University \& Shandong Academy of Medical Sciences, 619 Changcheng Road, Taian 271016, People's Republic of China

Yun He

\section{References}

1. Song $Y$, Zhang $Y$, Han Z, Xu W, Xiao J, Wang X, et al. Genetic recombination in fast-spreading coxsackievirus A6 variants: a potential role in evolution and pathogenicity. Virus Evol. 2020;6:2. doi:10.1093/ve/veaa048.

2. Chen J, Han Z, Wu H, Xu W, Yu D, Zhang Y. A Large-Scale Outbreak of Echovirus 30 in Gansu Province of China in 2015 and Its Phylodynamic Characterization. Front Microbiol. 2020;11:1137.

3. Han Z, Song Y, Xiao J, Jiang L, Huang W, Wei H, et al. Genomic epidemiology of coxsackievirus A16 in mainland of China, 2000-18. Virus Evol. 2020;6:2. doi:10.1093/ve/veaa084. 
4. Suresh S, Forgie S, Robinson J. Non-polioEnterovirus detection with acute flaccid paralysis: A systematic review. J Med Virol. 2018;90:3-7.

5. Simmonds P, Gorbalenya AE, Harvala H, Hovi T, Knowles NJ, Lindberg AM, et al. Recommendations for the nomenclature of enteroviruses and rhinoviruses. Arch Virol. 2020;165:793-7.

6. Fairweather D, Stafford KA, Sung YK. Update on coxsackievirus B3 myocarditis. Curr Opin Rheumatol. 2012;24:401-7.

7. Fu X, Mao L, Wan Z, Xu R, Ma Y, Shen L, et al. High proportion of coxsackievirus B3 genotype A in hand, foot and mouth disease in Zhenjiang, China, 2011-2016. Int J Infect Dis. 2019;87:1-7.

8. Han Z, Zhang Y, Huang K, Wang J, Tian H, Song Y, et al. Two Coxsackievirus B3 outbreaks associated with hand, foot, and mouth disease in China and the evolutionary history worldwide. BMC Infect Dis. 2019;19:466.

9. Simonen-Tikka ML, Pflueger M, Klemola P, Savolainen-Kopra C, Smura T, Hummel S, et al. Human enterovirus infections in children at increased risk for type 1 diabetes: the Babydiet study. Diabetologia. 2011;54:2995-3002.

10. Rose NR. Viral myocarditis. Curr Opin Rheumatol. 2016;28:383-9.

11. Garmaroudi FS, Marchant D, Hendry R, Luo H, Yang D, Ye X, et al. Coxsackievirus B3 replication and pathogenesis. Future Microbiol. 2015;10:629-53.

12. Guo H, Li Y, Liu G, Jiang Y, Shen S, Bi R, et al. A second open reading frame in human enterovirus determines viral replication in intestinal epithelial cells. Nat Commun. 2019;10:4066.

13. Zhang Y, Zhu Z, Yang W, Ren J, Tan X, Wang Y, et al. An emerging recombinant human enterovirus 71 responsible for the 2008 outbreak of hand foot and mouth disease in Fuyang city of China. Virol J. 2010;7:94.

14. Zhang Y, Wang D, Yan D, Zhu S, Liu J, Wang H, et al. Molecular evidence of persistent epidemic and evolution of subgenotype B1 coxsackievirus A16-associated hand, foot, and mouth disease in China. J Clin Microbiol. 2010;48:619-22.

15. Zhang $Y$, Wang J, Guo W, Wang H, Zhu S, Wang D, et al. Emergence and transmission pathways of rapidly evolving evolutionary branch $\mathrm{C} 4 \mathrm{a}$ strains of human enterovirus 71 in the Central Plain of China. PLoS One. 2011;6:e27895.

16. Kyriakopoulou Z, Pliaka V, Amoutzias GD, Markoulatos P. Recombination among human non-polio enteroviruses: implications for epidemiology and evolution. Virus Genes. 2015;50:177-88.

17. Mandary M, Poh C. Changes in the EV-A71 Genome through Recombination and Spontaneous Mutations: Impact on Virulence. Viruses. 2018;10:320.

18. Kroneman A, Vennema H, Deforche K, Avoort HVD, Peñaranda S, Oberste MS, et al. An automated genotyping tool for enteroviruses and noroviruses. J Clin Virol. 2011;51:121-5.

19. Rambaut A, Lam TT, Max Carvalho L, Pybus OG. Exploring the temporal structure of heterochronous sequences using TempEst (formerly Path-O-Gen). Virus Evol. 2016;2:1. doi:10.1093/ve/vew007. 
20. Drummond AJ, Rambaut A. BEAST: Bayesian evolutionary analysis by sampling trees. BMC Evol Biol. 2007;7:214.

21. Salminen MO, Carr JK, Burke DS, McCutchan FE. Identification of breakpoints in intergenotypic recombinants of HIV type 1 by bootscanning. AIDS Res Hum Retroviruses. 1995;11:1423-5.

22. Stålhanske PO, Lindberg AM, Pettersson U. Replicase Gene of Coxsackievirus B3. J Virol. 1984;51:742-6.

23. Kandolf R, Hofschneider PH. Molecular cloning of the genome of a cardiotropic Coxsackie B3 virus: Full-length reverse-transcribed recombinant cDNA generates infectious virus in mammalian cells. Proc Natl Acad Sci U S A. 1985;82:4818-22.

24. Clemmer DI, Li F, Le Blanc DR, Fox JP. An outbreak of subclinical infection with coxsackievirus B3 in southern lousiana. Am J Epidemiol. 1966;83(1):123-9.

25. Rossouw E, Tsilimigras CW, Schoub BD. Molecular Epidemiology of a Coxsackievirus B3 outbreak. J Med Virol. 1991;34:165-71.

26. Nakayama T, Urano T, Osano M, Hayashi Y, Sekine S, Ando T, Makinom S. Outbreak of herpangina associated with Coxsackievirus B3 infection. Pediatr Infect Dis J. 1989;8:495-8.

27. Dechkum N, Pangsawan Y, Jayavasu C, Saguanwongse S. Coxsackie B virus infection and myopericarditis in Thailand, 1987-1989. Southeast Asian J Trop Med Public Health. 1998;29:273-6. PMID: 9886111.

28. Wu Z, Du J, Zhang T, Xue Y, Yang F, Jin Q. Recombinant human coxsackievirus B3 from children with acute myocarditis in China. J Clin Microbiol. 2013;51:3083-6.

29. Tseng F, Huang H, Chi C, Lin T, Liu C, Jian J, et al. Epidemiological Survey of Enterovirus Infections Occurring in Taiwan Between 2000 and 2005: Analysis of Sentinel Physician Surveillance Data. J Med Virol. 2007;79:1850-60.

30. Tao Z, Song Y, Li Y, Liu Y, Jiang P, Lin X, et al. Coxsackievirus B3, Shandong Province, China, 19902010. Emerg Infect Dis. 2012;18:1865-7.

31. Wong AH, Lau CS, Cheng PKC, Ng AYY, Lim WWL. Coxsackievirus B3-associated aseptic meningitis: An emerging infection in Hong Kong. J Med Virol. 2011;83:483-9.

32. Prill MM, Dahl RM, Midgley CM, Chern SW, Lu X, Feikin DR, et al. Severe Respiratory Illness Associated With Rhinovirus During the Enterovirus D68 Outbreak in the United States, August 2014November 2014. Clin Infect Dis. 2018;66:1528-34.

33. Baertl S, Pietsch C, Maier M, Hönemann M, Bergs S, Liebert UG. Enteroviruses in Respiratory Samples from Paediatric Patients of a Tertiary Care Hospital in Germany. Viruses. 2021;13:882.

34. Machado RS, de Sousa IP Jr, Monteiro JC, Ferreira JL, Dos Santos Alves JC, Tavares FN. Detection and identification of enteroviruses circulating in children with acute gastroenteritis in Pará State, Northern Brazil (2010-2011). Virol J. 2020;17:156.

35. Chansaenroj J, Tuanthap S, Thanusuwannasak T, Duang-in A, Klinfueng S, Thaneskongtong N, et al. Human enteroviruses associated with and without diarrhea in Thailand between 2010 and 2016. 
PLoS One. 2017;12(7):e182078.

36. Lee CJ, Huang YC, Yang S, Tsao KC, Chen CJ, Hsieh YC, et al. Clinical Features of Coxsackievirus A4, B3 and B4 Infections in Children. PLoS One. 2014;9:e87391.

37. Duong V, Mey C, Eloit M, Zhu H, Danet L, Huang Z, et al. Molecular epidemiology of human enterovirus 71 at the origin of an epidemic of fatal hand, foot and mouth disease cases in Cambodia. Emerg Microbes Infect. 2016;5:e104-9.

38. Smets K, Keymeulen A, Wollants E, Lagrou K, Van Ranst M, Padalko E. Detection of enteroviral RNA on Guthrie card dried blood of a neonate with fatal Coxsackie B3 myocarditis on day 17. J Clin Virol. 2008;42:207-10.

39. Calderón Kl, Díaz-de Cerio M, Otero A, Muñoz-Almagro C, Rabella N, Martínez-Rienda l, et al. Molecular epidemiology of coxsackievirus B3 infection in Spain, 2004-2014. Arch Virol. 2016;161:1365-70.

40. Andréoletti L, Ventéo L, Douche-Aourik F, Canas F, de la Grandmaison GL, Jacques J, et al. Active Coxsackieviral B Infection Is Associated With Disruption of Dystrophin in Endomyocardial Tissue of Patients Who Died Suddenly of Acute Myocardial Infarction. J Am Coll Cardiol. 2007;50:2207-14.

41. Zhang W, Lin X, Jiang P, Tao Z, Liu X, Ji F, et al. Complete genome sequence of a coxsackievirus B3 recombinant isolated from an aseptic meningitis outbreak in eastern China. Arch Virol. 2016;161:2335-42.

42. Mirand A, Henquell C, Archimbaud C, Chambon M, Charbonne F, Peigue-Lafeuille H, et al. Prospective Identification of Enteroviruses Involved in Meningitis in 2006 through Direct Genotyping in Cerebrospinal Fluid. J Clin Microbiol. 2008;46:87-96.

43. Mirand A, Henquell C, Archimbaud C, Chambon M, Charbonne F, Peigue-Lafeuille H, et al. Prospective of Enteroviruses Involved in Meningitis in 2006 through Direct Genotyping in Cerebrospinal Fluid. J Clin Microbiol. 2008;46:87-96.

44. Ai J, Xie Z, Liu G, Chen Z, Yang Y, Li Y, et al. Etiology and prognosis of acute viral encephalitis and meningitis in Chinese children: a multicentre prospective study. BMC Infect Dis. 2017;17:494.

45. Fernandez-Garcia MD, Kebe O, Fall AD, Ndiaye K. Identification and molecular characterization of non-polio enteroviruses from children with acute flaccid paralysis in West Africa, 2013-2014. Sci Rep. 2017;7:3808.

46. Bingjun T, Yoshida H, Yan W, Lin L, Tsuji T, Shimizu H, et al. Molecular typing and epidemiology of non-polio enteroviruses isolated from Yunnan Province, the People's Republic of China. J Med Virol. 2008;80:670-9.

47. Sadeuh-Mba SA, Kavunga-Membo H, Joffret M, Yogolelo R, Endegue-Zanga MC, Bessaud M, et al. Genetic landscape and macro-evolution of co-circulating Coxsackieviruses A and Vaccine-derived Polioviruses in the Democratic Republic of Congo, 2008-2013. PLoS Negl Trop Dis. 2019;13:e7335.

48. Yan K, Yang J, Qian Q, Xu D, Liu H, Wei L, et al. Pathogenic Role of an IL-23/YסT17/Neutrophil Axis in Coxsackievirus B3-Induced Pancreatitis. J Immunol. 2019;203:3301-12. 
49. Viskari H, Knip M, Tauriainen S, Huhtala H, Veijola R, llonen J, et al. Maternal Enterovirus Infection as a Risk Factor for Type 1 Diabetes in the Exposed Offspring. Diabetes Care. 2012;35:1328-32.

50. Ronellenfitsch S, Tabatabai J, Böttcher S, Diedrich S, Frommhold D, Schubert-Bast S, et al. First report of a Chinese strain of coxsackie B3 virus infection in a newborn in Germany in 2011: a case report. J Med Case Rep. 2014;8:164.

51. Lukashev AN, Lashkevich VA, Ivanova OE, Koroleva GA, Hinkkanen AE, llonen J. Recombination in circulating Human enterovirus $B$ : independent evolution of structural and non-structural genome regions. J Gen Virol. 2005;86:3281-90.

52. Lukashev AN. Role of recombination in evolution of enteroviruses. Rev Med Virol. 2005;15:157-67.

53. Kim J, Jeon E, Lim B, Kim S, Chung S, Kim J, et al. Immunogenicity of a DNA vaccine for coxsackievirus B3 in mice: protective effects of capsid proteins against viral challenge. Vaccine. 2005;23:1672-9.

54. Shen H, Liu T, Luo Y, Shao S, Deng X, Wang H. Echovirus plays major roles in the natural recombination of Coxsackievirus B3. J Med Virol. 2018;90:377-82.

55. Wang H, Qian Y, Qian C, Dai C, Shen H. Two Natural Recombination gave rise to the Coxsackievirus B3 GV that triggered outbreaks in China in 2006-2012. Infect Dis Now. 2021;51:81-5.

\section{Figures}

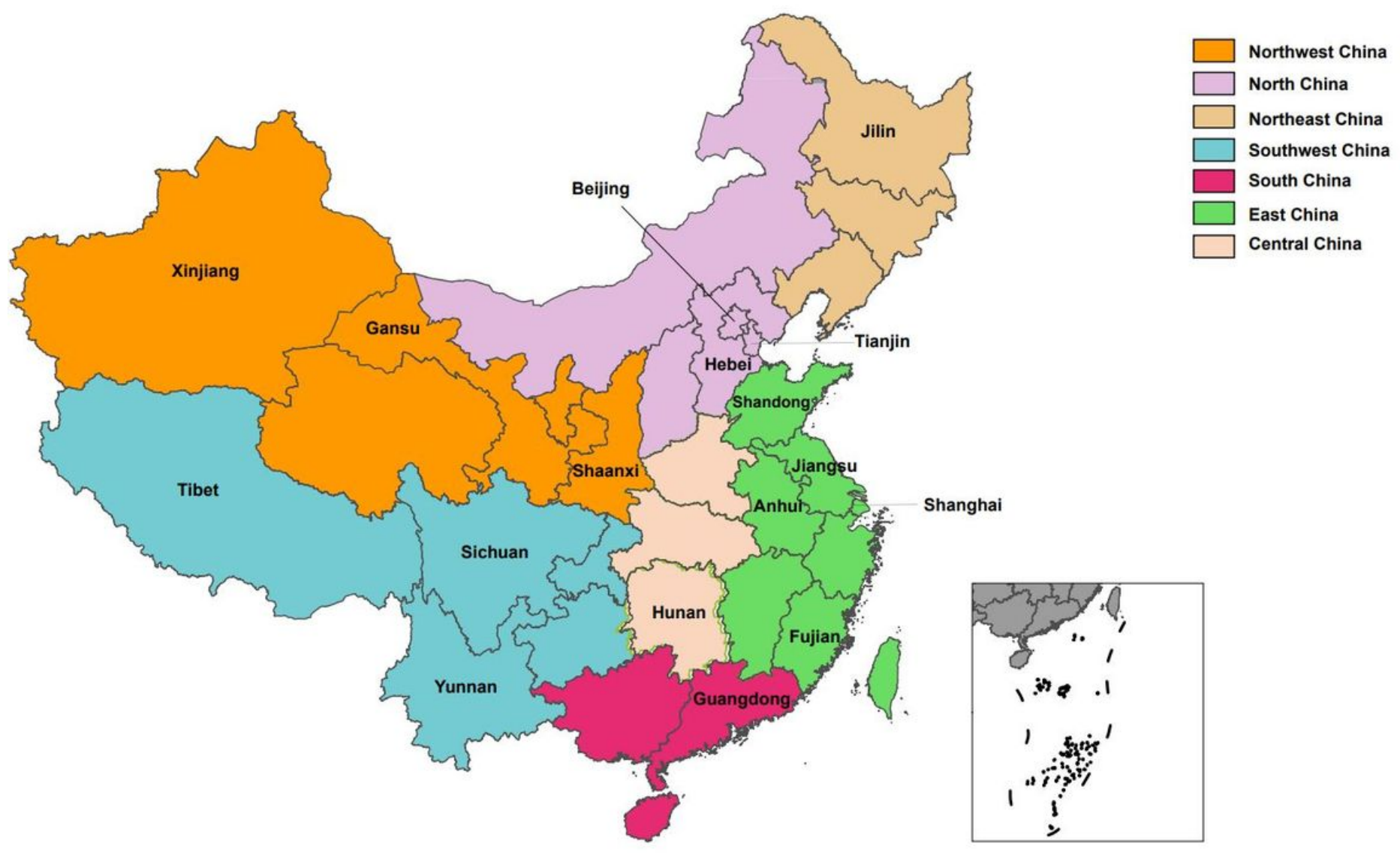

Page $18 / 23$ 
Figure 1

The geographical distribution whole genome of CVB3 sequences in mainland China.

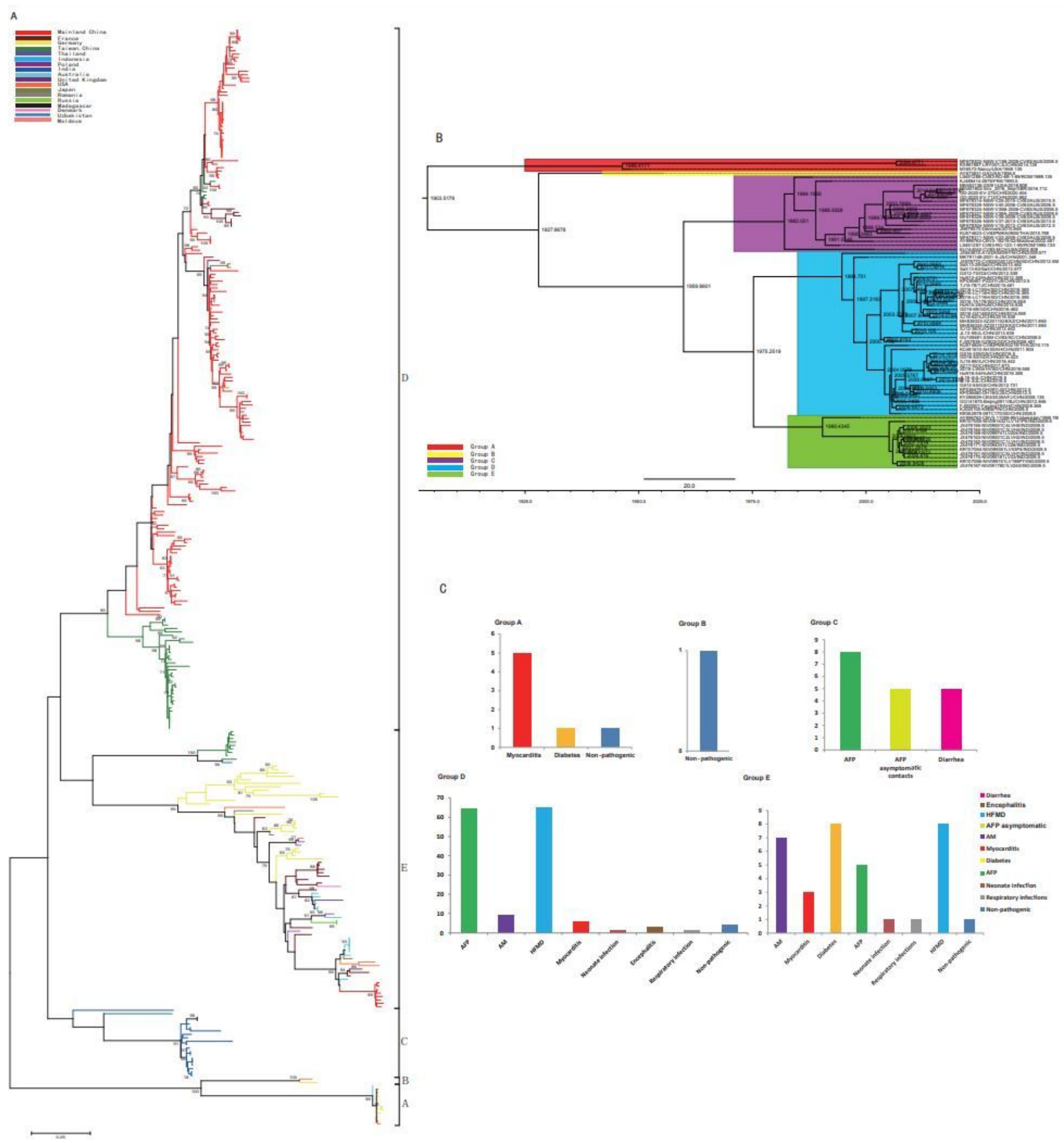

Figure 2

The genetic diversity and clinical manifestations distribution of CVB3 worldwide as determined in this study. (A) The maximum likelihood phylogenetic tree based on the entire VP1 genome of 319 CVB3 
genome sequences for group division. (B) The maximum clade credibility (MCC) phylogenetic tree based on the entire P1 coding region of 77 CVB3 strains around the world. (C) The distribution of diseases caused by CVB3 in different groups. The numbers of disease cases in each group are indicated by in the bar chart.

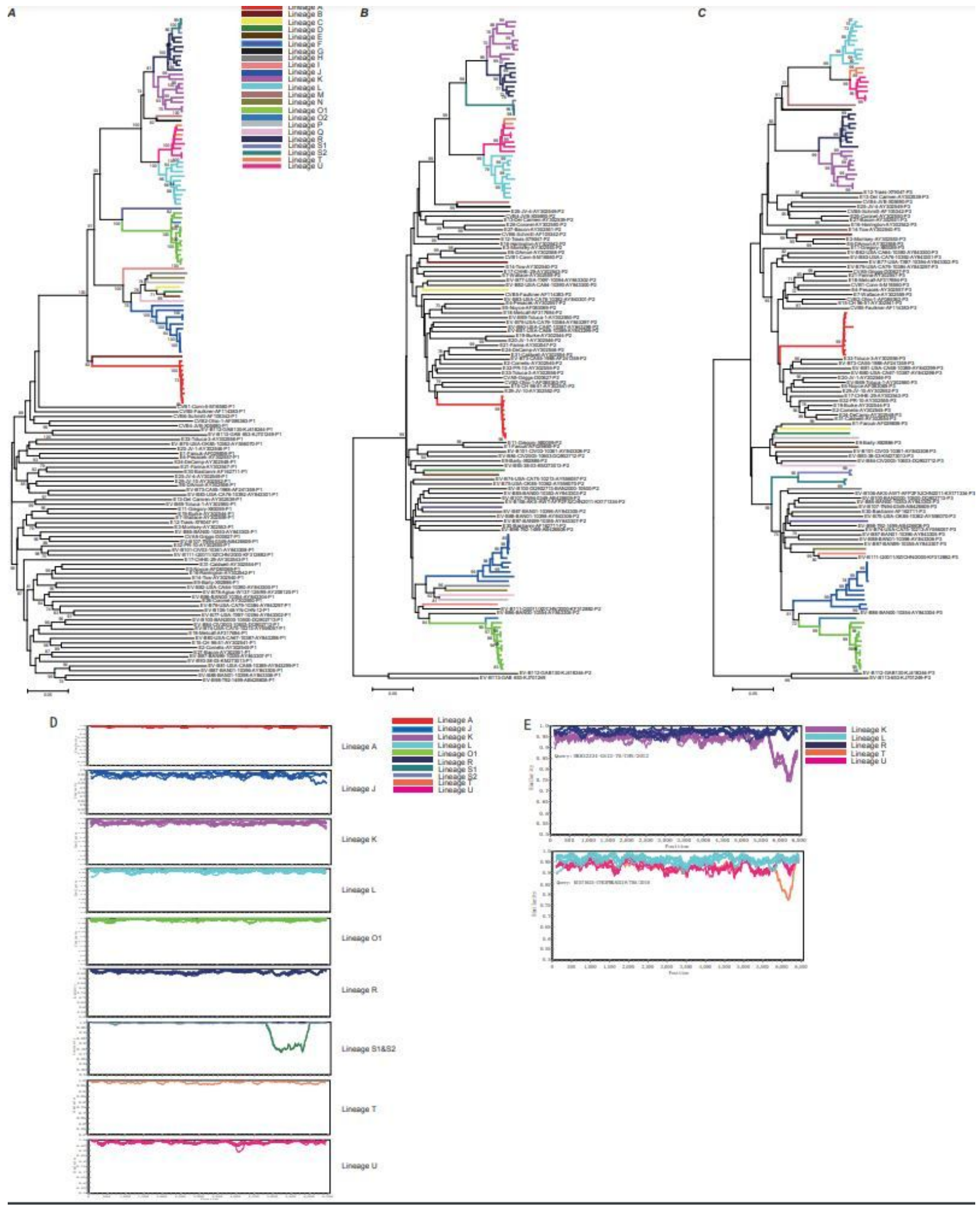

Figure 3 
Neighbor-joining phylogenetic trees of 84 global CVB3 constructed based on the coding regions. (A) P1 capsid region. (B) P2 non-structural region. (C) P3 non-structural region. The lineages are differentiated by distinct colors. (D) Intra-lineage pairwise similarity comparisons based on ORF1 sequences using sliding window nucleotide similarity analysis with a 200-nt window moving in 20-nt steps; similarity was not calculated for lineages containing only one sequence. (E) Inter-lineage pairwise similarity comparison of main lineages circulating in mainland China based on ORF1 sequences using sliding window nucleotide similarity analysis with a 200-nt window moving in 20-nt steps.

A
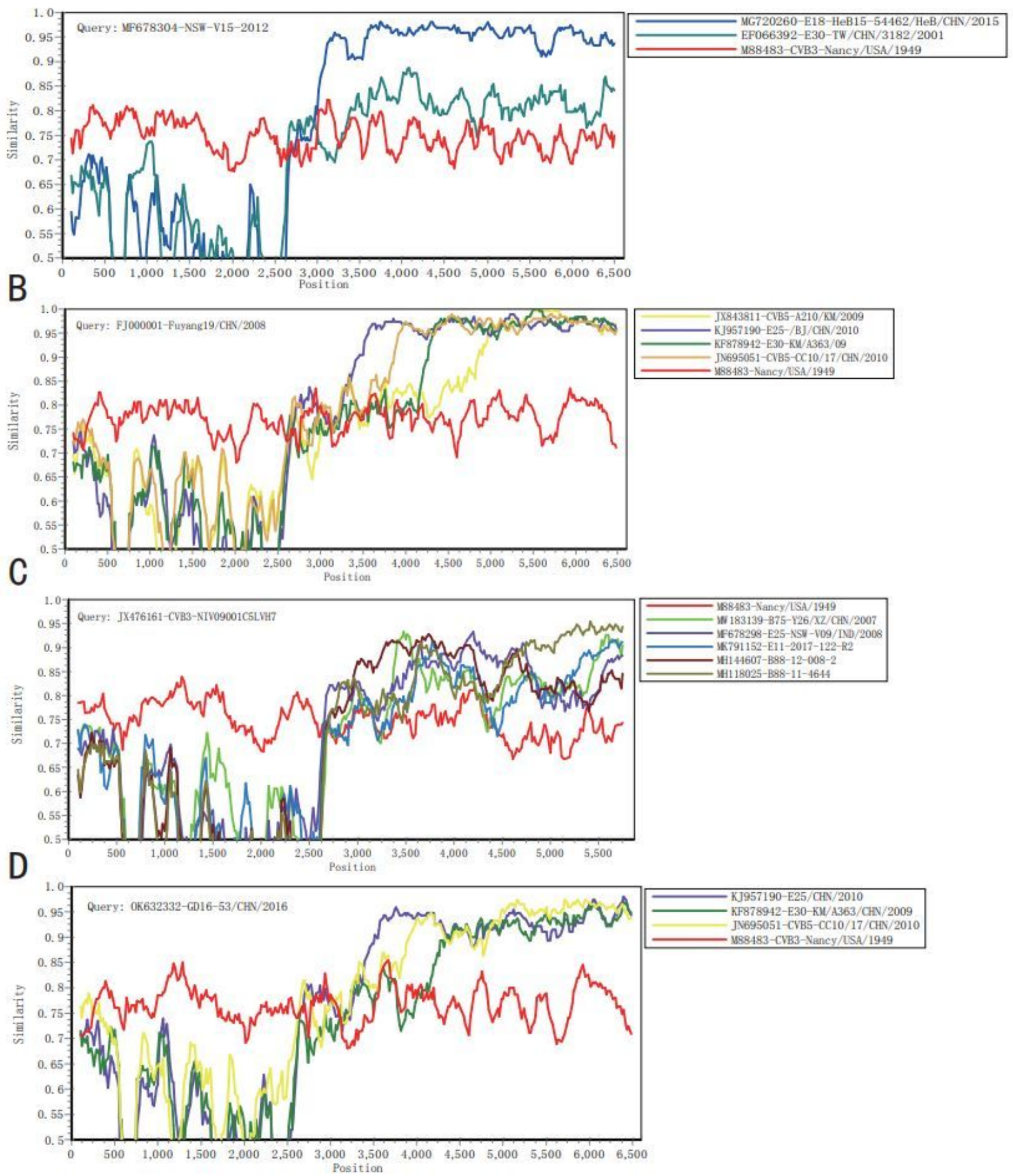
Figure 4

Similarity plots were constructed for detecting potential recombination detection among the screened EV$B$ strains and (A) lineage $J$, (B) lineage $L$, (C) lineage 01, and (D) lineage $U$.
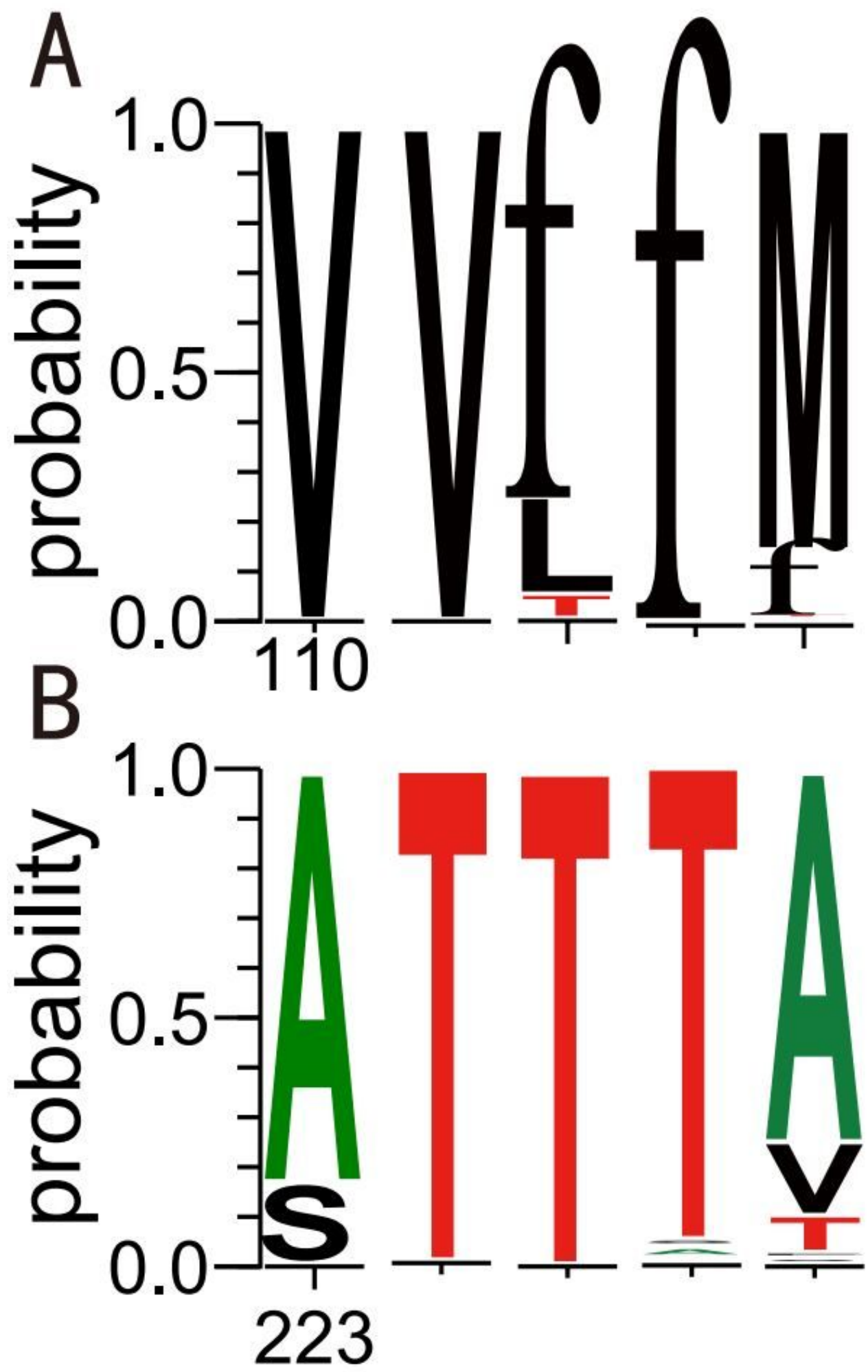

Figure 5

The amino acid positions of 110 (A) and 223 (B) in the VP1 region among 5 group CVB3 strains. 


\section{Supplementary Files}

This is a list of supplementary files associated with this preprint. Click to download.

- Additionalfile1TableS1.docx 